Cathy Helen Wardle, Beyond Écriture féminine. Repetition and Transformation in the Prose Writing of Jeanne Hyvrard

\title{
Elisa Borghino
}

\section{(2) OpenEdition \\ Journals}

\section{Edizione digitale}

URL: http://journals.openedition.org/studifrancesi/8085

DOI: $10.4000 /$ studifrancesi.8085

ISSN: 2421-5856

\section{Editore}

Rosenberg \& Sellier

\section{Edizione cartacea}

Data di pubblicazione: 1 juillet 2009

Paginazione: 444-445

ISSN: 0039-2944

\section{Notizia bibliografica digitale}

Elisa Borghino, "Cathy Helen Wardle, Beyond Écriture féminine. Repetition and Transformation in the Prose Writing of Jeanne Hyvrard», Studi Francesi [Online], 158 (LIII | II) | 2009, online dal 30 novembre 2015, consultato il 12 janvier 2021. URL: http://journals.openedition.org/studifrancesi/8085 ; DOI: https://doi.org/10.4000/studifrancesi.8085

Questo documento è stato generato automaticamente il 12 janvier 2021.

\section{cc) (†) $\odot$}

Studi Francesi è distribuita con Licenza Creative Commons Attribuzione - Non commerciale - Non opere derivate 4.0 Internazionale. 


\title{
Cathy Helen Wardle, Beyond Écriture féminine. Repetition and Transformation in the Prose Writing of Jeanne Hyvrard
}

\author{
Elisa Borghino
}

\section{NOTIZIA}

CATHY HELEN WARDLE, Beyond Écriture féminine. Repetition and Transformation in the Prose Writing of Jeanne Hyvrard, London, Maney Publishing for the Modern Humanities Research Association, 2007, pp. 145.

1 Il volume, nato da una tesi di dottorato, approfondisce l'opera e la figura di Jeanne Hyvrard, autrice francese contemporanea nata a Parigi nel 1945. Attraverso un'attenta analisi delle opere hyvrardiane, Cathy Helen Wardle propone un percorso articolato in sei capitoli correlati da introduzione e conclusione. Numerosi e puntuali non solo $\mathrm{i}$ riferimenti alle opere di Jeanne Hyvrard, ma anche ai testi critici di Jacques Derrida, Julia Kristeva e Philippe Lejeune, solide fondamenta all'approccio di tipo psicoanalitico e matricentrico voluto da Wardle. I temi trattati spaziano dal rapporto tra logos, chaos e tierce pensée al ricordo della madre, dal ritorno alle origini ai giochi di parole, dalla visione del testo come labirinto alla trasformazione del sé.

2 Autrice di romanzi, racconti, poesie, saggi e testi sperimentali, Jeanne Hyvrard pubblica il primo romanzo, Les Prunes de Cythère, nel 1975. È proprio intorno ad esso che ruota l'introduzione, centrata su reazioni critiche e intertestualità. Il romanzo, che già mostra i segni caratterizzanti della scrittura hyvrardiana, è costruito sulla base del patto autobiografico e attinge all'esperienza relazionale con la madre, intesa come nodo fondamentale della vita dell'autrice. Proprio la relazione madre-figlia diventerà 
uno dei temi ricorrenti, insieme a quello della donna pazza, del ricordo e della ripetizione.

Come spiega l'autrice nell'introduzione, l'intenzione primaria è di togliere al lavoro di Hyvrard l'etichetta di écriture féminine, pur mantenendo la consapevolezza dell'identità sessuale nei suoi pensieri e nella sua scrittura. Jeanne Hyvrard vede infatti nella letteratura un mezzo alternativo per esprimere la complessa realtà che la circonda. Lo stretto legame tra letteratura e filosofia è mostrato sin dalle prime righe dell'introduzione, che riportano una citazione tratta da A bord du marais della stessa Hyvrard: «La littérature montre ce que la philosophie ne sait pas encore démontrer» (p. 1).

4 Al centro dell'opera di Wardle sono gli schemi attraverso cui Hyvrard ripete e trasforma la mitologia - in modo particolare i miti di Persefone e della Creazione, quest'ultimo proponendosi come metodo di analisi della scrittura hyvrardiana nonché le ripetizioni intertestuali interne ed esterne, che coinvolgono il lettore in un processo di costante reinterpretazione in relazione con le nozioni di linearità e di caos della vita reale di Jeanne Hyvrard. «Le chaos» scrive Hyvrard in Canal de la Toussaint, «est l'organisation en train de se faire, l'organisation en train de s'accomplir, l'organisation même, le monde» (p. 134). 